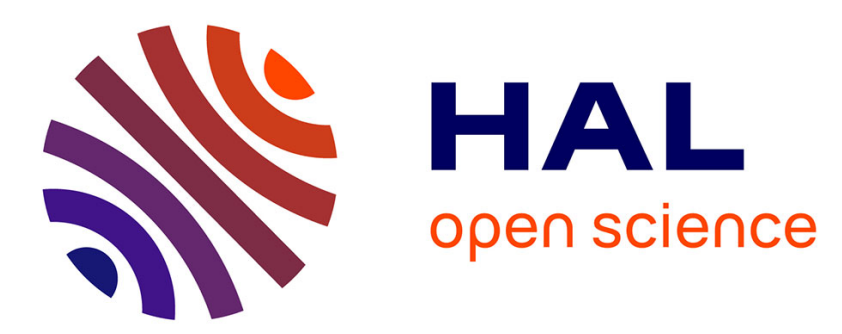

\title{
Legitimate domain of a Newtonian behavior for thermal nanoimprint lithography
}

Hubert Teyssedre, Pierre Gilormini, Stefan Landis, Gilles Regnier

\section{To cite this version:}

Hubert Teyssedre, Pierre Gilormini, Stefan Landis, Gilles Regnier. Legitimate domain of a Newtonian behavior for thermal nanoimprint lithography. Microelectronic Engineering, 2013, 110, pp.215-218. 10.1016/j.mee.2013.03.169 . hal-00842918

\section{HAL Id: hal-00842918 https://hal.science/hal-00842918}

Submitted on 9 Jul 2013

HAL is a multi-disciplinary open access archive for the deposit and dissemination of scientific research documents, whether they are published or not. The documents may come from teaching and research institutions in France or abroad, or from public or private research centers.
L'archive ouverte pluridisciplinaire HAL, est destinée au dépôt et à la diffusion de documents scientifiques de niveau recherche, publiés ou non, émanant des établissements d'enseignement et de recherche français ou étrangers, des laboratoires publics ou privés. 


\title{
Legitimate domain of a Newtonian behavior for thermal nanoimprint lithography
}

\author{
H. Teyssèdre ${ }^{\mathrm{a}, \mathrm{b}, *}$, P. Gilormini ${ }^{\mathrm{a}}$, S. Landis ${ }^{\mathrm{c}}$, G. Régnier ${ }^{\mathrm{a}}$ \\ ${ }^{a}$ PIMM, Arts et Métiers ParisTech, 151 bvd de l'Hôpital, 75013 Paris, France \\ ${ }^{b}$ Silsef, 74160 Archamps, France \\ ${ }^{c}$ CEA/LETI, 17 rue des Martyrs, 38054 Grenoble, France
}

\begin{abstract}
Nanoimprint lithography is an efficient way to reproduce nanostructures down to 20 nanometers in sub-micrometer polymeric films. To optimize this process, simulation using a Newtonian behavior is a cheap and efficient way to predict the polymer flow in micro and nano size cavities. This behavior is nevertheless limited to flows with shear rates below a critical value that can be determined with standard rheology measurements. We have investigated the validity domain of this behavior to simulate thermal NIL. This domain of validity is composed of two uncoupled functions, one for the material properties and the mean pressure applied to the pattern, and one for the geometry considered. The latter function has been determined with numerical simulations using the natural element method. It is demonstrated that knowing the mean applied pressure, the critical shear rate, and the viscosity of the material we are able to determine, depending on stamp geometry, if shear-thinning may or may not occur during an imprinting process.
\end{abstract}

Keywords: Imprint simulation, Newtonian behavior, Shear-thinning

\section{Introduction}

Nanoimprint lithography (NIL) is a process where nanometric patterns are engraved into a very thin polymer film. In the variant considered here, thermal nanoimprint, the polymer is spin coated on a silicon wafer before imprint at temperature above the glass transition temperature. The surfaces involved are of the order of hundreds of square centimeters to allow for the simultaneous imprint of a very large number of patterns. Although thermal

\footnotetext{
${ }^{*}$ Corresponding author: Phone: +33617612571

Email address: hubert.teyssedre@cea.fr (H. Teyssèdre)
}

published in Microelectronic Engineering, vol. 110, pp. 215-218 (2013) 
NIL has shown a great potential to overcome the limitations of conventional photolithographic methods, this process is still prone to defects, either localized due to the uncontroled polymer flow through cavities [1], or long range defects due to mold bending [2]. To optimize this process, numerical and analytical tools for the simulation of polymer flow have been developed based on continuum mechanics with different types of behavior. Experiments have shown that viscoelasticity model is prone to be the most suitable behavior in general cases [3]. In particular for an imprint temperature close to the glass transition temperature, both experiments [4] and simulations [5] have shown that the elastic effects are predominant. In other cases, shear-thinning can be used [6] as a first step of equations simplification, but this requires to solve non-linear systems.Thus, in a more restricted set of cases, the Newtonian behavior has been used as a cheap and efficient way to predict the polymer flow in micro and nano size cavities. This behavior has shown a remarkable capability to simulate the reflow of nano size structures to measure the viscosity in sub-micrometer polymeric films $[7,8]$. Nevertheless, assuming the material obeys more likely a Carreau-Yasuda law [9], this behavior is limited to flows with shear rates lower than a critical value that can be determined with standard rheology measurements.

In this paper, we analyze in which extent the Newtonian behavior can be used regarding pattern geometry, applied pressure and material parameters. First, we will state the mechanical problem and present the rheological properties of the polystyrene used in this study. In section 3 we present the critical area within the polymer below the stamp protrusion affected by shear-thinning, and the normalization of the maximum shear rate over the flow. Numerical simulations are reported in section 4, which allow finally to discuss the validity of the Newtonian behavior regarding the pattern aspect ratio and the consequences of shear-thinning on the process.

\section{Problem statement}

Nanoimprint lithography can be used to reproduce a variety of patterns, but simple lines are very frequently used to demonstrate capabilities of this technique, and to test models. One advantage of line patterns is the twodimensional flow involved, which allows the study of a mere cross section. Moreover, the study can be limited to configurations where the polymer does not touch the recessed parts of the stamp, and a single period when the pattern is periodic, and even to half a period for symmetry reasons, as shown in figure 1. As it has been shown in a previous study [10], considering the initial state of the imprint or a current one is approximatively the same. So only the initial state is considered. The pattern is characterized by its 
pitch $(W)$ and width $(L)$, and the thickness of the polymer $(h)$ defines the last geometric parameter of the problem.

A no-slip boundary condition is assumed between the polymer and the mold, and no pressure is applied on the free surface of the material. Moreover, as the purpose of the present study is to explore the validity domain of the Newtonian model, surface tension is neglected, although it may play an important role at the nano scale. A consequence of these assumptions is that the process parameter can be indifferently either the downward velocity of the mold $v_{d}$ or the mean pressure applied on the mold $\bar{p}=F / L$, since these two quantities are proportional when the fluid is Newtonian. Finally, inertial effects and gravity can be neglected in microflows and nanoflows of viscous liquids [11], where extremely low Reynolds numbers are involved, and a Stokes flow without body forces is considered here, consequently.

\section{Critical area to be excluded and shear rate normalization}

The addressed problem is similar to the indentation of a rigid flat base punch with sharp square corners into a soft incompressible elastic surface [12], by interpreting the displacement field as a velocity field and the shear modulus as a viscosity. It has been shown that pressure and shear stress reach infinite values under the corners in such a problem [12]. These singularities are also demonstrated in numerical simulations, where the thinner the mesh is, the higher the maximal computed values of pressure and shear rate are under the corners. These infinite values illustrate the limits of a linear behavior to describe this indentation problem. Consequently, shear-thinning is expected in these regions for real materials, even at the lightest load. In this case we can nevertheless expect the shear-thinning effects to be limited to a small volume fraction of the polymer, with small consequences on the results elsewhere. Therefore, the solution calculated with a Newtonian behavior everywhere is assumed to be valid except in the vicinity of the pattern corners, and we define a critical area as a disc centered on the corner with a radius $\varepsilon$, as presented in figure 1 . The shear rate will be studied outside this area and is expected to reach a maximum on the disc boundary. The radius will be compared to the width $L$ of the pattern in order to give an estimation of the validity of the Newtonian solution. The ratio $\varepsilon / L$ defines the fraction of polymer where shear-thinning is likely to appear, and its value depends on the accuracy needed for the solution. In this paper we use a $\varepsilon / L$ ratio of 4\%. An optimum value could be determined by matching simulations with experimental results.

Figure 2 presents our rheological measurements on a $280 \mathrm{~kg} / \mathrm{mol}$ polystyrene at 170 C. It has been purchased from Sigma Aldrich (9003-53-6/182427), and 
is named PS280 in this paper. The viscosity $\eta$ of the polymer is plotted versus the shear rate $\dot{\gamma}^{e q}$. A Carreau-Yasuda behavior with a soft transition from a constant viscosity $\eta_{0}$, corresponding to the Newtonian plateau, to a power-law dependence is observed and is described by the following equation:

$$
\eta\left(\dot{\gamma}^{e q}\right)=\frac{\eta_{0}}{\left(1+\left(\dot{\gamma}^{e q} / \dot{\gamma}_{0}\right)^{a}\right)^{\frac{1-n}{a}}}
$$

with the following parameters fitting our rheometric measurements on PS280 at $170 \mathrm{C}: \eta_{0}=1.2 \times 10^{4}$ Pa.s, $\dot{\gamma}_{0}=2.7 \mathrm{~s}^{-1}, a=0.9$ and $n=0.13$. The transition is characterized by the critical shear rate $\dot{\gamma}_{0}$ defined by the intersection of the two asymptotes, as shown in figure 2. The Newtonian behavior is assumed to be valid as long as we have $\dot{\gamma}^{e q}<\dot{\gamma}_{0}$ in the polymer, where $\dot{\gamma}^{e q}$ is the equivalent shear rate defined as:

$$
\dot{\gamma}^{e q}=\sqrt{2 \mathbb{D}: \mathbb{D}}
$$

where $\mathbb{D}$ is the strain rate tensor.

The Newtonian approach remains one of the major hypotheses of the simulation and it remains valid as long as $\dot{\gamma}_{\text {max }}^{e q}<\dot{\gamma}_{0}$ is verified, where $\dot{\gamma}_{\text {max }}^{e q}$ denotes the maximum shear rate found in the flow. This criterion is usually difficult to check experimentally as no in situ real time measurement technique at the nanoscale is available. It can only be verified in simulations and can be widely investigated using a non-dimensional approach. The width $L$ is thus chosen as a reference length. Since the behavior is linear, the velocity field is proportional to the imposed velocity of the mold $v_{d}$, and so are the strain rates. The force on the mold is proportional to the viscosity $\eta_{0}$ and to the mold velocity, and so is the mean pressure on the mold. Thus we can write:

$$
\dot{\gamma}_{e q}^{\max }=\frac{v_{d}}{L} \cdot \Lambda\left(\frac{h}{L}, \frac{W}{L}\right) \quad \bar{p}=\eta_{0} \cdot \frac{v_{d}}{L} \cdot \Phi\left(\frac{h}{L}, \frac{W}{L}\right)
$$

where $\Lambda$ and $\Phi$ are unknown functions that depend on the non-dimensional geometric parameters only. We then define the reference shear rate $\dot{\gamma}_{\text {ref }}=$ $\bar{p} / \eta_{0}$ as the ratio of the mean pressure on the mold and viscosity, and the condition to be fulfilled can be rewritten as

$$
\frac{\dot{\gamma}_{e q}^{\max }}{\dot{\gamma}_{r e f}}=\frac{\Lambda}{\Phi}\left(\frac{h}{L}, \frac{W}{L}\right)<\frac{\dot{\gamma}_{0}}{\dot{\gamma}_{r e f}} .
$$

In this expression, the left hand term depends on the non-dimensional geometry only. It can be determined by computing $\dot{\gamma}_{e q}^{\max }$ and $\bar{p}$ during an imprint for a reference material and a set of geometric parameters, and normalization allows to use it for other materials. Once the ratio $\Lambda / \Phi=\dot{\gamma}_{e q}^{\max } / \dot{\gamma}_{\text {ref }}$ has 
been determined, it should be compared to the right hand term calculated from the mean pressure $\bar{p}$ that will be applied on the mold, the Newtonian viscosity $\eta_{0}$ and the critical shear rate $\dot{\gamma}_{0}$. This formula has also the advantage not to depend on temperature: using the WLF law [13] for the imprint of amorphous polymers over the glass transition temperature leads to

$$
\log \frac{\eta_{0}(T)}{\eta_{W L F}}=a(T) \quad \log \frac{\dot{\gamma}_{0}(T)}{\dot{\gamma}_{W L F}}=-a(T)
$$

with $a(T)$ the shift parameter, $\eta_{W L F}$ and $\dot{\gamma}_{W L F}$ being the reference parameters of the WLF law. These relations point out the fact that the higher the temperature is, the less viscous the material is and the wider the Newtonian plateau, and that the product $\dot{\gamma}_{0} \cdot \eta_{0} / \bar{p}=\dot{\gamma}_{0} / \dot{\gamma}_{\text {ref }}$ does not depend on temperature.

To summarize, the above criterion has the advantage of uncoupling the geometric parameters from the material and process ones, and to be valid regardless the imprint temperature. The critical point is the computation of $\dot{\gamma}_{e q}^{\max } / \dot{\gamma}_{\text {ref }}$ which is developed in the next section.

\section{C-NEM simulations}

To determine $\dot{\gamma}_{e q}^{\max } / \dot{\gamma}_{r e f}$, an in-house developed software [14] has been used. The code uses the natural element method (NEM) to simulate viscous flows. The principle of the NEM has been introduced by Traversoni [15], but the first application of this meshless method is due to Braun and Sambridge [16], who considered two-dimensional Stokes flows. The method has been applied later to solid mechanics by Sukumar et al. [17], whose work promoted the method and stimulated subsequent developments. As an extension of the NEM, the C-NEM variant that we use here has been developed for non convex boundaries by Yvonnet et al. [18]. In contrast with the finite element method, the C-NEM employs natural neighbor interpolation without fixed connectivity and has low sensitivity to mesh distortion, among other advantages, which makes it a promising method for the simulation of fluid flows.

In this work, the C-NEM is used with a mixed formulation to simulate planar incompressible Stokes flows for thermal NIL, with the stamp modeled as a rigid body, neglecting gravity and capillary effects. Our first goal is to estimate $\dot{\gamma}_{e q}^{\max }$ in the vicinity of the corner, outside the critical area. The velocity field is first computed with the simulation tool over all the domain, which allows us to determine the equivalent shear rate at each node. As expected the highest values are obtained for the nodes in contact with the mold, and they increase close to the stamp feature corner. The maximal 
value retained is the equivalent shear rate at the intersection of the critical area and the mold, given by a linear interpolation of the neighboring values.

Then we compute the reaction force on the mold and divide it by the width $L$ to obtain $\bar{p}$, which is less sensitive to the number of nodes as it is an integrated quantity. Two sets of $W / L(2$ and 4$)$ were computed for $h / L$ ranging from $5 \times 10^{-3}$ to 3 .

The computation of the left hand term $\dot{\gamma}_{e q}^{\max } / \dot{\gamma}_{r e f}$ of inequality (4) is presented in figure 3 for $\varepsilon / L=4 \%$. Increasing this ratio will decrease the computed maximum shear rate as presented in figure 4 . The curves demonstrate three regimes. In figure 3 , for $h / L<5 \times 10^{-2}$, the solution does not depend on $W / L$, which means the quantity of polymer below the cavity does not influence the flow below the pattern. This shows that the amount of polymer under the cavity does not oppose significantly the lateral flow for ultra-thin layers. As a consequence, the strain rate field for a given $h / L$ value is unchanged whether we are in the initial state $(t=0)$ or in a current imprinted state $(t>0)$. Over an important $h / L$ value $(1.5$ for $W / L=2$ and 2.5 for $W / L=4), \dot{\gamma}_{e q}^{\max } / \dot{\gamma}_{\text {ref }}$ is constant and the solution is close to the hotembossing configuration in this regime, that we will call the hot-embossing

plateau. In between these two regimes, the maximum value of $\dot{\gamma}_{e q}^{\text {max }} / \dot{\gamma}_{\text {ref }}$ depends on the $W / L$ ratio. Depending on the $\dot{\gamma}_{e q}^{\max } / \dot{\gamma}_{r e f}$ ratio, we will show in the next section how shear thinning may play a significant role in the process.

\section{Application and discussion}

As an application, consider for instance a typical pattern with $L=$ $150 \mathrm{~nm}, W=600 \mathrm{~nm}, h_{0}=300 \mathrm{~nm}$, which leads to $h / L=2$ and $W / L=4$. Figure 4 shows the corresponding normalized maximum shear rate for two sizes of the critical area, $\varepsilon / L=4 \%$ and $40 \%$. For the process condition, a very low pressure is chosen in order to observe the transitions between Newtonian and shear-thinning regimes, with $\bar{p}=0.8$ bar. Using the material parameters obtained on the PS280, namely a critical shear rate $\dot{\gamma}_{0}=2.7 \mathrm{~s}^{-1}$ and a Newtonian viscosity $\eta_{0}=1.2 \times 10^{4}$ Pa.s, the upper limit $\dot{\gamma}_{0} / \dot{\gamma}_{\text {ref }}$ takes the value of 0.4 . In figure 4 , imprint process starts with high $h / L$ value. At $h / L=2$ the normalized maximum shear rate keeps under the horizontal 0.4 value shown as a horizontal line, down to point $\mathrm{A}(h=180 \mathrm{~nm})$. Therefore, inequation (4) is satisfied and the Newtonian regime is dominant. When imprint proceeds from point A to point $\mathrm{C}(h=15 \mathrm{~nm})$, the curve is above the critical value, the inequation is violated, and shear-thinning cannot be neglected. The Newtonian solution is then no more relevant, with a maximum error reached at point B for $h=45 \mathrm{~nm}$. The Newtonian behavior is acceptable again after point $\mathrm{C}$, for $h<15 \mathrm{~nm}$, as the $\varepsilon / L=4 \%$ curve 
recover a lower value than $\dot{\gamma}_{0} / \dot{\gamma}_{\text {ref }}$. This illustrates that the Newtonian behavior can be used to evaluate either the imprint time or velocity reliably at the beginning and end of the imprint process only. As a comparison the curve for $\varepsilon / L=40 \%$ is also plotted. As expected this curve is below the previous one as it allows shear-thinning to occur in a larger area, and within our experimental parameters it is still cross by the horizontal line.

It may also be noticed that increasing the pressure on the stamp will increase $\dot{\gamma}_{r e f}$ and decrease the upper limit, consequently. Shear-thinning would thus appear sooner in the process, or even from the very beginning if the upper limit goes lower than the hot-embossing plateau of the normalized maximum shear rate curve.

Between the two regimes cited above where the Newtonian model is valid, one should rather consider a non-linear vicous model, such as the CarreauYasuda law used in this paper. Moreover, a visco-elastic model should be preferred if the imprint is performed close to the glass temperature, in order to take into account the elastic recovery [4].

\section{Conclusion}

We have investigated the validity domain of the Newtonian behavior to simulate thermal NIL. It has been shown that the simple case of a sharp cornered pattern would induce shear-thinning in the polymer flow even for the lightest load. A critical area has been defined to quantify the proportion of polymer that undergoes shear-thinning and helps to establish the domain of validity of the linear behavior. It is defined by two uncoupled functions, one depending on material properties and on the mean pressure applied to the pattern, and the other depending on geometric parameters only. The latter function has been determined with numerical simulations based on the natural element method. We have demonstrated that knowing the mean applied pressure, the critical shear rate, and the viscosity of the material, it is possible to determine, depending of the stamp geometry, if shear-thinning may or may not occur during an imprint. It appears that in most common configurations a Newtonian behavior is relevant at the beginning and at the end of the process.

\section{Acknowledgements}

The authors are grateful to L. Illoul for making his C-NEM routines available. Support from the Agence Nationale de la Recherche through project SINCRONE is acknowledged. 


\section{References}

[1] S. Landis, N. Chaix, D. Hermelin, T. Leveder, C. Gourgon, Microelectronic Engineering 84 (2007) 940-944.

[2] S. Landis, N. Chaix, C. Gourgon, C. Perret, T. Leveder, Nanotechnology 17 (2006) 2701-2709.

[3] B. S. O. Graham L. W. Cross, Richard M. Langford, J. B. Pethica, in: Materials Research Society 2005 (Ed.), 2004 MRS Fall Meeting, volume 841, p. R1.6.

[4] H.-C. Scheer, N. Bogdanski, M. Wissen, T. Konishi, Y. Hirai, Journal of Vacuum Science \& Technology B: Microelectronics and Nanometer Structures 23 (2005) 2963.

[5] N. W. Kim, K. W. Kim, H.-C. Sin, Microelectronic Engineering 85 (2008) 1858-1865.

[6] H. D. Rowland, A. C. Sun, P. R. Schunk, W. P. King, Journal of Micromechanics and Microengineering 15 (2005) 2414-2425.

[7] T. Leveder, S. Landis, L. Davoust, N. Chaix, Microelectronic Engineering 84 (2007) $928-931$.

[8] E. Rognin, S. Landis, L. Davoust, Physical Rewiew E 84 (2011).

[9] P. Carreau, Transactions os the Society of Rheology 16 (1972) 99.

[10] H. Teyssedre, P. Gilormini, G. Régnier, International Polymer Processing 28 (2012) 72-78.

[11] G. Karniadakis, A. Beskok, N. R. Aluru, Microflows And Nanoflows: Fundamentals And Simulation, Springer, 2005.

[12] K. L. Johnson, K. L. Johnson, Contact Mechanics, Cambridge University Press, 1987.

[13] M. L. Williams, R. F. Landel, J. D. Ferry, Journal of the American Chemical Society 77 (1955) 3701-3707.

[14] H. Teyssedre, P. Gilormini, Journal of Non-Newtonian Fluid Mechanics, in the press (2012). 
[15] L. Traversoni, in: Blain, WR and Katsifarakis, KL (Ed.), Free Surface Flow and Hydraulic Software, pp. 291-297. 5th International Conference on Hydraulic Engineering Software (HYDROSOFT 94), PORTO CARRAS, GREECE, SEP, 1994.

[16] J. Braun, M. Sambridge, Nature 376 (1995) 655-660.

[17] N. Sukumar, B. Moran, T. Belytschko, International Journal For Numerical Methods in Engineering 43 (1998) 839.

[18] L. P. Yvonnet J., Ryckelynck D., C. F., Int. J. Numer. Meth. Engng. 60 (2004) 1451-1474. 


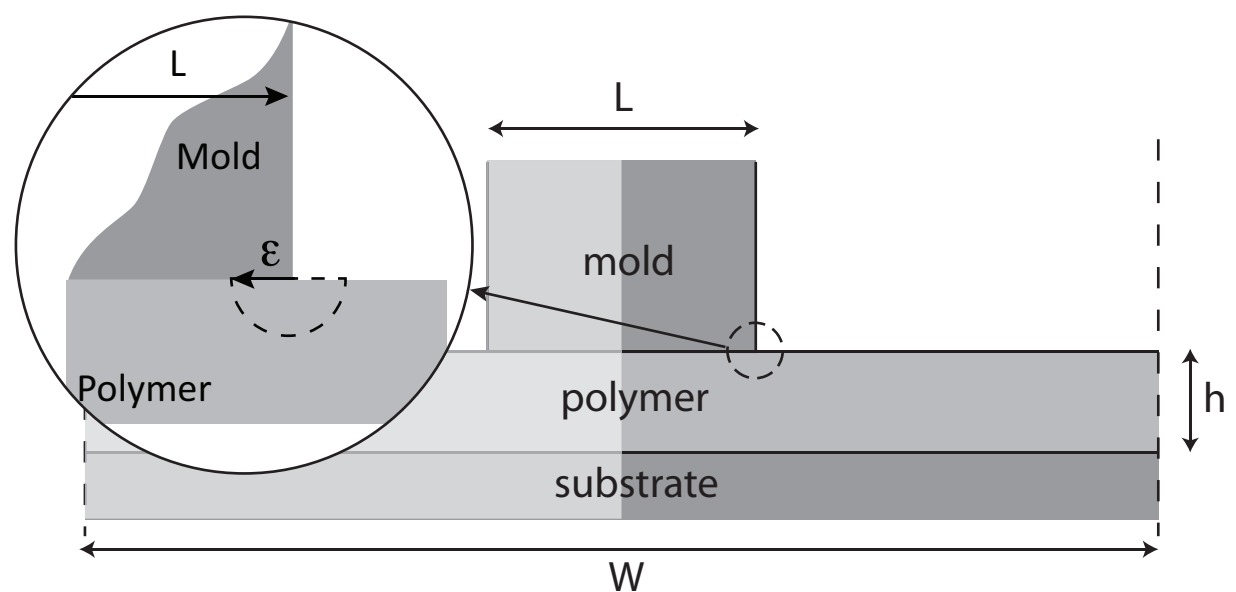

Figure 1: Schematic description of one period considered for simulation. The minimal part that need being considered is the darker gray shades. The definition of the critical area $\varepsilon$ around the corner is also presented. 


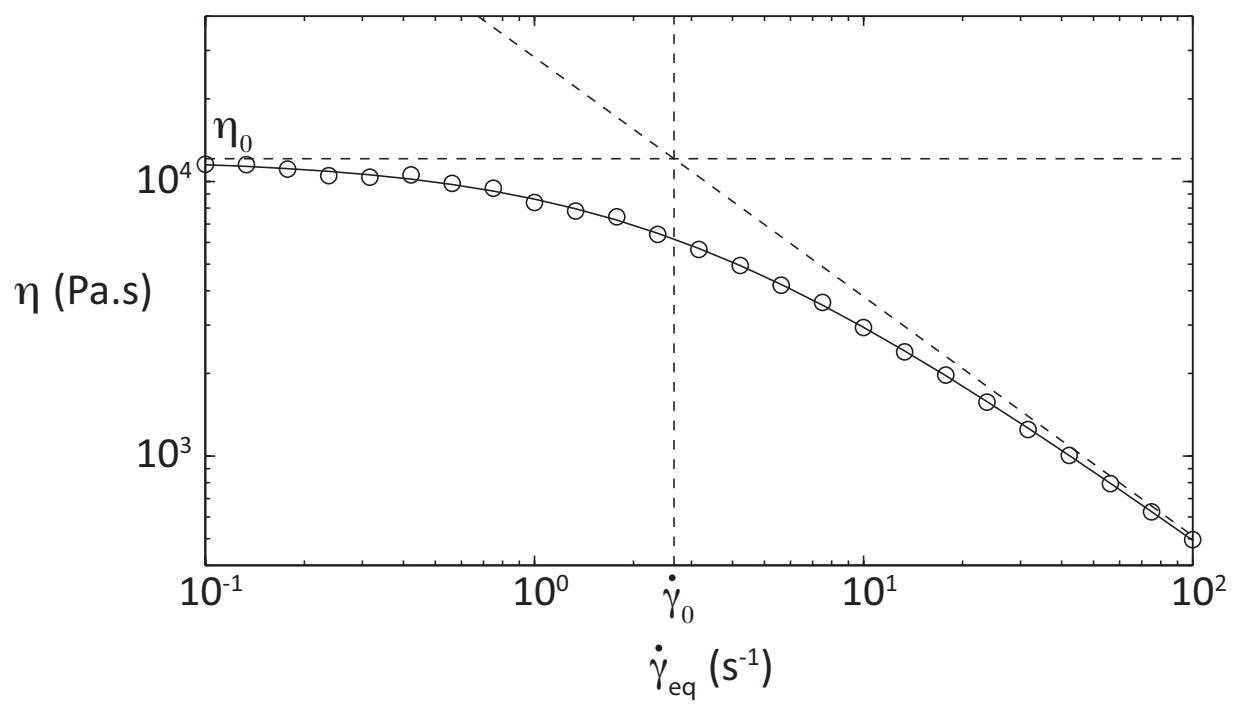

Figure 2: Viscosity of a PS280 at $170 \mathrm{C}$ versus shear rate. Experimental data (circles), Carreau-Yasuda fit (solid curve), asymptotes (horizontal and oblique dashed lines), and critical shear rate $\dot{\gamma}_{0}$ (vertical dashed line) are plotted. 


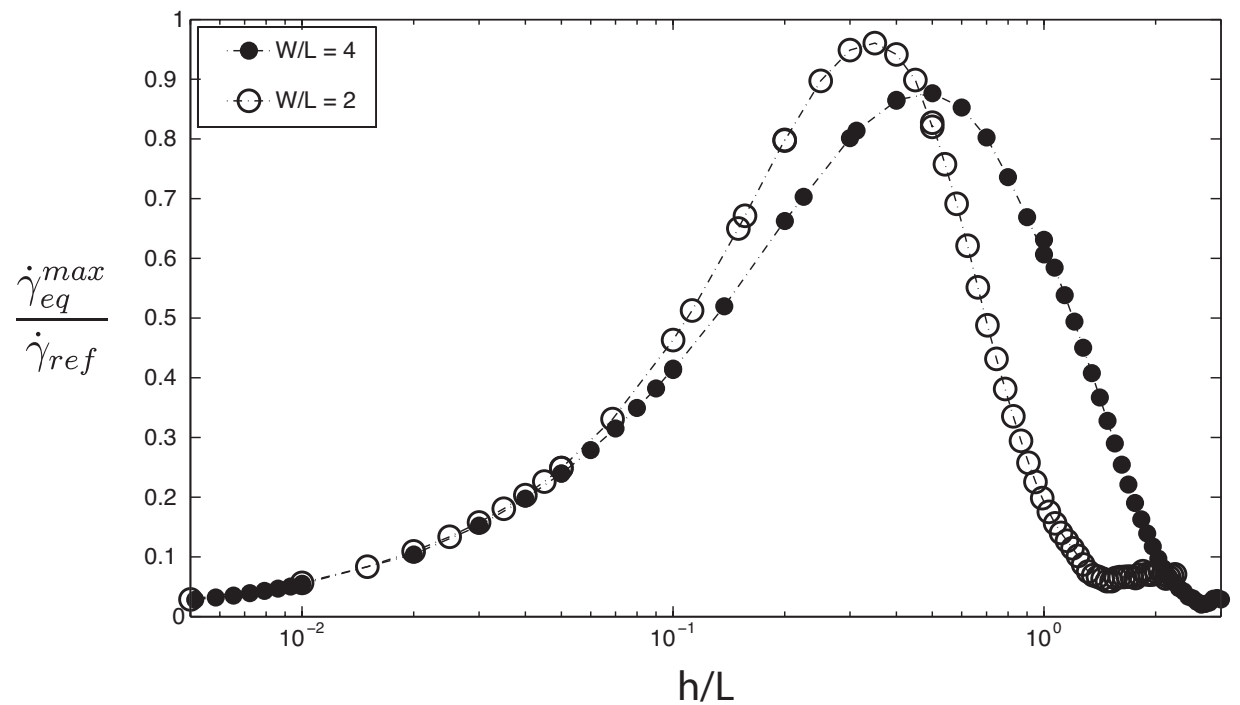

Figure 3: Normalized maximum shear rate computed at the beginning of imprint, as a function of the non-dimensional initial thickness $h / L$ and pitch $W / L$ for a critical area defined by $\varepsilon / L=4 \%$. 


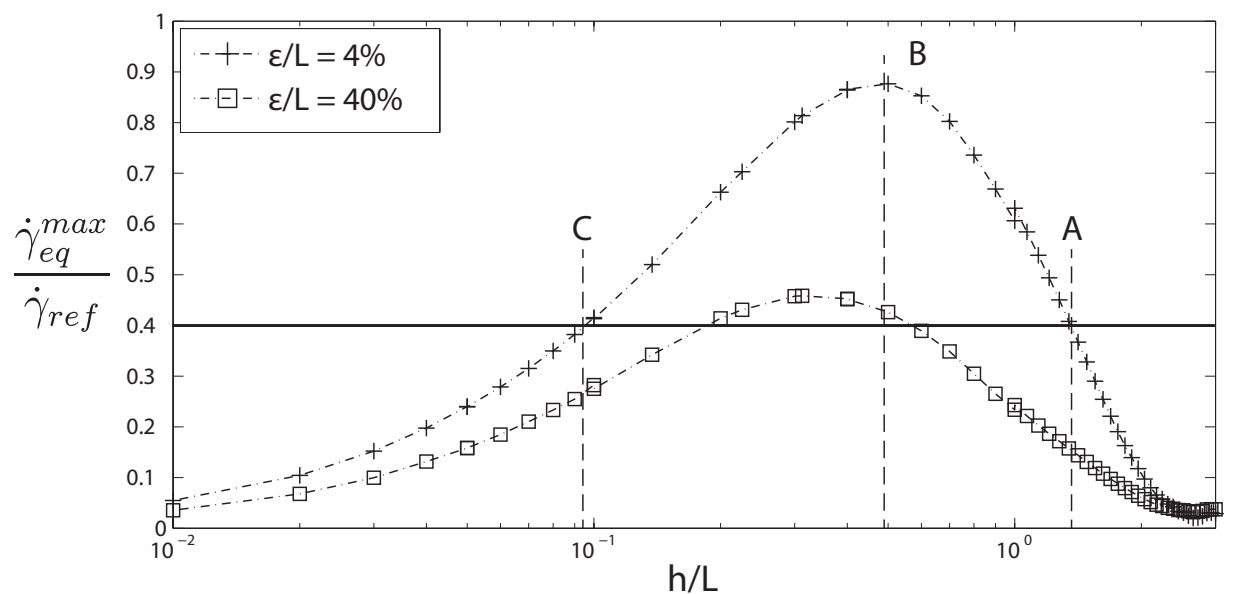

Figure 4: Legitimate domain of a Newtonian behavior as a function of the non-dimensional initial thickness $h / L$, with $W / L=4$, for two values of the critical area size $\varepsilon / L$. The horizontal line is determined from the process condition and material parameters. 